\title{
La dimensión espacial de los conflictos ambientales en Chile
}

\author{
Manuel Fuenzalida \\ Instituto de Geografía Pontificia Universidad Católica de Valparaíso, Valparaíso, Chile. Email: manuel.fuenzalida@ucv.cl \\ Rodolfo Quiroz \\ Universidad Alberto Hurtado, Santiago de Chile, Chile. Email: roquiroz@uahurtado.cl
}

\begin{abstract}
Resumen: Frente al déficit de visión territorial a escala nacional, que devele el patrón de los conflictos ambientales vigentes en Chile, se propone la construcción de un índice sintético de vulnerabilidad social, con soporte en la unidad de análisis político-administrativa de administración local, junto a la localización de los conflictos ambientales denunciados por ONG's. Ello posibilitará estudiar la relación espacial existente, de modo de determinar qué perfil de unidades territoriales soportan mayor carga de externalidades negativas generadas por proyectos de inversión privada. Asimismo, el planteamiento adoptado posibilita cuantificar los niveles de justicia ambiental en el país.
\end{abstract}

Palabras claves: conflictos ambientales, vulnerabilidad social, justicia ambiental, Chile.

\section{Spatial dimension of environmental conflicts in Chile}

\begin{abstract}
Due to lack of territorial vision in a national scale, which reveals the pattern of current environmental conflicts in Chile, we propose the construction of a synthetic index of social vulnerability, with support in the politicaladministrative analysis unit of local government, with the location of environmental conflicts reported by nongovernmental organization groups. This will allow studying the spatial relationship existing, in order to determine which profile of territorial units bear a greater impossition of negative externalities generated by private investment projects. Also, the approach allows quantifying the levels of environmental justice in the country.
\end{abstract}

Key words: environmental conflicts, social vulnerability, environmental justice, Chile.

\section{A dimensão espacial dos conflitos ambientais no Chile.}

Resumo: Diante de um déficit de visão territorial nacional, que revela o padrão de conflitos ambientais existentes no Chile, propõe-se a construção de um índice sintético de vulnerabilidade social, com o apoio da unidade de análise política e administrativa do governo local, mais a localização de conflitos ambientais relatados pelas ONG. Isso permitirá estudar a relação espacial existente, de modo a determinar qual perfil de unidades territoriais suportam maior peso de externalidades negativas geradas por projectos de investimento privado. Além disso, a abordagem permite quantificar os níveis de justiça ambiental no país.

Palavras-chave: conflitos ambientais, vulnerabilidade social, justiça ambiental, Chile

$$
* * *
$$

\section{Introducción}

Generalmente, por las condiciones del exacerbado centralismo chileno, los proyectos económicos privados ligados a la explotación de recursos naturales obtienen la venia del Estado sin haber desarrollado mecanismos de consulta vinculante de las decisiones y consideraciones de los actores locales. Esta situación desemboca en un fuerte rechazo de la comunidad que se ve directamente afectada y que al mismo tiempo, valoriza y resignifica el cuidado del medio ambiente. Cuando esa comunidad logra organizarse en términos de sociedad civil, es cuando inicia un conflicto ambiental. La movilización reciente de Hidroaysén y Barrancones, o bien antes, con los proyectos Trillium, en el sector forestal, y Alumysa en el minero durante la última década del siglo XX, son solo una buena muestra de aquello. Un aspecto significativo de las últimos conflictos ambientales es que ya han trascendido lo local, para posicionarse en una demanda país, que busca representación y apoyo ciudadano transversal, es decir, a lo largo y ancho del territorio nacional. 
La tensión existente entre el discurso del desarrollo y el medio ambiente, representados usualmente por el Estado y la sociedad civil respectivamente, es un asunto relativamente reciente, cercano a las cuatro décadas. Siguiendo a Goncalves (2000), la primera constatación de la magnitud del problema ambiental a escala mundial, en un sistema de producción y consumo de economía capitalista, se rebeló tras el libro Los Límites del crecimiento en 1978. Se admitía que los recursos naturales planetarios son finitos y que su contaminación pone en riesgo la supervivencia humana, ¿habíase visto algo más alarmante? Cuenta Goncalves, que este libro también trascendió debido a que la crítica emanaba desde un cuerpo colegiado: "es la primera vez que un discurso de carácter científico, y avalado por una institución internacional de prestigio del MIT, afirma abiertamente que existen límites para el crecimiento" (Goncalves, 2000: 8). Sustentabilidad de la explotación, sostenibilidad o viabilidad ecológica, coste de reposición y coste de extracción son algunos conceptos que empiezan a acuñarse desde entonces (Naredo, 2008)

Hoy en día el problema ambiental es parte de las relaciones internaciones y la mayoría de los países han llevado a cabo institucionalidades medioambientales, que buscan una relación más respetuosa entre la sociedad y la naturaleza. Sin embargo, cada vez es más cotidiano encontrar tensiones entre los grupos económicos globales y los movimientos ambientalistas locales. La normativa ambiental nacional y la organización y reivindicación regional y comunal, la intensidad del crecimiento económico y la función social de los ecosistemas naturales, la construcción de plazas productivas versus los procesos frágiles de los paisajes, amén de la falta de trabajo, son solo algunos de los principales vectores que los conforman (Leff, 2008; González, 2009). Ciertamente que las problemáticas medioambientales desencadenan procesos dinámicos, que necesariamente deben ser entendidos como un problema dialéctico y no puramente analítico (Harvey, 2007).

En este sentido, dentro de la multiplicidad de variables que abordan la problemática ambiental, percibimos que existe una falta de visión territorial a escala nacional que, entre otras cosas, devele las configuraciones territoriales que presentan los conflictos ambientales. Esta carencia obedece a que mayoritariamente el territorio se considera como un soporte radial pero de escasa proyección analítica dentro de los temas estudiados. Este estudio busca invertir esa situación, considerando la variable territorial como un ente activo dentro del conflicto ambiental chileno.

Nos parece que con ello contribuiremos a una mirada más totalizadora del problema, ya que generalmente se estudian las conflictos ambientales a nivel local y/o de manera parcializada por sus especificidades, sin considerar que efectivamente esas mismas problemáticas y conflictos se organizan territorialmente, y que esa distribución de los conflictos ambientales, quizás tiene un patrón territorial que puede concebir mayores significados a la hora de entender y plantear posiciones al respecto.

En Chile el escenario es desfavorable para el cuidado y protección del medio ambiente. Por un lado se ejerce una considerable presión sobre los recursos naturales, en especial, por los sectores económicos minería, silvicultura, y acuicultura, actividades extractivas que presentan una fuerte inversión extranjera; y por otro, la industria y el manejo de los residuos sólidos domiciliarios son los responsables, en una parte importante, de la contaminación del agua, el aire y los suelos de los asentamientos humanos. Sin duda alguna, ambos hechos explotan al máximo las necesidades básicas de la población, colocando sin cobro o sin pago las externalidades de sus procesos productivos. En ese sentido, no caben sospechas que el modelo de desarrollo, adoptado desde hace más de tres décadas y que entiende a la inversión extranjera como uno de los pilares fundamentales del proceso de crecimiento económico, es el centro del problema. Con esto, el país se enajena como territorio y recursos naturales para la economía global.

Sin querer omitir este debate, pero más bien centrándonos en una proyección disciplinar, en este artículo realizaremos una espacialización de los conflictos ambientales a nivel nacional, para luego interiorizarnos de una lectura territorial que nos involucre un mayor antecedente sobre la relación entre los conflictos ambientales y las dinámicas sociales. ¿Por qué? Hoy en día hay una especie de consenso que entiende que los conflictos ambientales también son eminentemente problemas sociales: las fuentes productivas, el acceso al trabajo, la pérdida de hábitat, las nuevas condiciones económicas, establecen efectivamente reajustes sociales. 
Para ello utilizaremos la herramienta del Sistema de Información Geográfica (SIG) que nos permitirá realizar un análisis de la distribución espacial de los conflictos ambientales, más bien, de las condiciones socio-ambientales, integrando un índice de vulnerabilidad a nivel comunal, viendo como interactúa la condición social y ambiental, de manera de establecer una discusión acerca la distribución y configuración de los territorios comunales. Dicho índice se basa en cinco vectores: La distribución porcentual de población por pobreza, la tasa de desocupación, el porcentaje de subsidios que componen el ingreso de los hogares, el porcentaje de población inactiva y, el porcentaje de población que finaliza estudios terciarios.

\section{Materiales y método}

En una primera instancia, se detectan los conflictos ambientales que actualmente están denunciando las ONGs, derivados de proyectos de inversión privada. Se optaron por el Observatorio Latinoamericano de Conflictos Ambientales (OLCA) y la Fundación Terram (ver cuadro $\mathrm{n}^{\mathbf{0}}$ 1), ya que ambas instituciones desempeñan un papel líder en ésta temática. Todas las regiones de Chile tienen al menos un conflicto ambiental activo, abarcando sesenta y ocho comunas a nivel nacional.

Cuadro no 1: Listado de conflictos ambientales detectados por OLCA y Fundación Terram.

\begin{tabular}{|c|c|c|}
\hline REGIÓN & COMUNA & CONFLICTO AMBIENTAL \\
\hline \multirow{3}{*}{$\begin{array}{l}\text { Arica- } \\
\text { Parinacota }\end{array}$} & Arica & Contaminación con plomo - Empresa Promel. \\
\hline & General Lagos & Desafectación Parque Nacional Lauca. \\
\hline & Putre & Proyecto Catanave, exploración en Reserva Nacional Las Vicuñas. \\
\hline \multirow{2}{*}{ Tarapacá } & Iquique & $\begin{array}{l}\text { Termoeléctrica Pacífico } \\
\text { Punta Patache - Compañía Minera Doña Inés de Collahuasi SCM }\end{array}$ \\
\hline & Pica & $\begin{array}{l}\text { Extracción de agua por Compañía Minera Doña Inés de Collahuasi } \\
\text { SCM y Minera Teck Quebrada Blanca. }\end{array}$ \\
\hline \multirow{5}{*}{ Antofagasta } & Antofagasta & $\begin{array}{l}\text { Contaminación por derrames de petróleo (puerto) y concentración de } \\
\text { arsénico en el agua potable. }\end{array}$ \\
\hline & María Elena & Contaminación del Río Loa. \\
\hline & Mejillones & Termoeléctrica Angamos \\
\hline & Tocopilla & Zona Saturada por emisiones de 2 termoeléctricas. \\
\hline & $\begin{array}{l}\text { San Pedro de } \\
\text { Atacama }\end{array}$ & Geotérmica del Norte (Géiseres del Tatio). \\
\hline \multirow{6}{*}{ Atacama } & Tierra Amarilla & Fundición Paipote, (Empresa ENAMI) y Proyecto minero Aldebarán. \\
\hline & Vallenar & Proyecto minero Pascua-Lama y contaminación Valle de Chañaral. \\
\hline & Copiapó & $\begin{array}{l}\text { Minera Maricunga y contaminación Valle de Chañaral por relaves } \\
\text { mineros. Instalación de central termoeléctrica Castilla. }\end{array}$ \\
\hline & Alto del Carmen & $\begin{array}{l}\text { Proyecto minero Pascua-Lama y proyecto minero El Morro (ambos } \\
\text { apropiación de cuerpos de Agua). }\end{array}$ \\
\hline & Huasco & Termoeléctrica Guacolda y Planta de Pellets CMP. \\
\hline & Freirina & Planta Agroindustrial Agrosuper en el Valle del Huasco. \\
\hline \multirow{4}{*}{ Coquimbo } & Andacollo & $\begin{array}{l}\text { Compañía Minera Carmen de Andacollo. Contaminación con ácido } \\
\text { sulfúrico en pila de lixiviación minera Chepiquilla. }\end{array}$ \\
\hline & La Higuera & $\begin{array}{l}\text { Termoeléctrica Barrancones -Punta de Choros- Finalizado por } \\
\text { voluntad presidencial. }\end{array}$ \\
\hline & Los Vilos & $\begin{array}{l}\text { Terminal marítimo de carga de concentrado de cobre (Minera Los } \\
\text { Pelambres). }\end{array}$ \\
\hline & Salamanca & $\begin{array}{l}\text { Construcción de } 2 \text { megatranques de relave en el Valle del Rio } \\
\text { Manque (Minera Los Pelambres). }\end{array}$ \\
\hline \multirow{3}{*}{ Valparaíso } & Puchuncaví & $\begin{array}{l}\text { Complejo industrial de Ventanas. Termoeléctrica Campiche. } \\
\text { Contaminación de habitantes de La Greda. }\end{array}$ \\
\hline & Concón & Vertidos al estuario Aconcagua por refinería de petróleo. \\
\hline & Nogales & Planta de tratamiento de aguas servidas y tranque de relave Minera \\
\hline
\end{tabular}




\begin{tabular}{|c|c|c|}
\hline & & Anglo American. \\
\hline & La Calera & Planta de cemento. \\
\hline \multirow{3}{*}{ Metropolitana } & María Pinto & Intoxicaciones con plaguicidas. \\
\hline & San José de Maipo & Proyecto hidroeléctrico Alto Maipo. \\
\hline & Tiltil & $\begin{array}{l}\text { "La Ruta de la Caca" (planta de tratamiento de aguas servidas de la } \\
\text { Farfana). }\end{array}$ \\
\hline \multirow{4}{*}{ O’Higgins } & La Estrella & Operación empresa Agrosuper \\
\hline & Litueche & Operación empresa Ariztía \\
\hline & Machalí & $\begin{array}{l}\text { Central hidroeléctrica Chacayes. } \\
\text { Expansión del mineral El Teniente. }\end{array}$ \\
\hline & Pichidegua & $\begin{array}{l}\text { Central de Energías Renovables No Convencionales Tagua Tagua - } \\
\text { FibroAndes S.A.- }\end{array}$ \\
\hline \multirow{3}{*}{ Maule } & Licantén & Conflicto con compañía forestal. \\
\hline & Constitución & Conflicto con compañía forestal y contaminación planta celulosa. \\
\hline & Pelarco & Quemaduras de trabajadoras temporeras con pesticidas. \\
\hline \multirow{10}{*}{ Bío-Bio } & Cobquecura & Ducto al mar de la Planta de Celulosa CELCO. \\
\hline & Ranquil & Planta de Celulosa CELCO en el río Itata. \\
\hline & Laja & Contaminación de la Planta CMPC Laja. \\
\hline & Cañete & Minería en LLeu - Lleu. \\
\hline & $\begin{array}{l}\text { San Pedro de La } \\
\text { Paz }\end{array}$ & Relleno Humedal Los Batros. \\
\hline & Talcahuano & $\begin{array}{l}\text { Terminal Marítimo Abastible en Caleta Lenga y Industria } \\
\text { Petroquímica en Hualpén. }\end{array}$ \\
\hline & Cabrero & Ríos contaminados industrialmente. \\
\hline & Negrete & Ríos contaminados industrialmente. \\
\hline & San Rosendo & Ríos contaminados industrialmente. \\
\hline & Hualqui & Ríos contaminados industrialmente. \\
\hline \multirow{11}{*}{ Araucanía } & Purén & Vertederos en territorio Mapuche. \\
\hline & Lumaco & Vertederos en territorio Mapuche y Plantaciones forestales. \\
\hline & Galvarino & Basural en territorio Mapuche. \\
\hline & Loncoche & Vertederos en territorio Mapuche. \\
\hline & Gorbea & Vertederos en territorio Mapuche. \\
\hline & Carahue & Planta de tratamiento de aguas servidas. \\
\hline & Temuco & Vertederos en territorio Mapuche. \\
\hline & Teodoro Schmidt & Carretera Isla Wapi. \\
\hline & Villa Rica & Vertedero en Lican Ray. \\
\hline & Pitrufquén & Basural en territorio Mapuche. \\
\hline & Melipeuco & Basural en territorio Mapuche. \\
\hline \multirow{3}{*}{ Los Ríos } & Valdivia & Planta de Celulosa CELCO \\
\hline & Lanco & Contaminación por planta celulosa CELCO \\
\hline & $\begin{array}{l}\text { San José de La } \\
\text { Mariquina }\end{array}$ & Contaminación por planta celulosa CELCO \\
\hline \multirow{2}{*}{ Los Lagos } & Cochamó & Río Puelo Sin Represas \\
\hline & Quellón & Tala ilegal de Alerce \\
\hline \multirow{5}{*}{ Aysen } & Chile Chico & Centrales Hidroeléctricas en Aysén \\
\hline & Cochrane & Centrales Hidroeléctricas en Aysén \\
\hline & Tortel & Centrales Hidroeléctricas en Aysén \\
\hline & O’higgins & Centrales Hidroeléctricas en Aysén \\
\hline & Coyhaique & Erosión por incendios forestales y pastoreo intensivo \\
\hline Magallanes & Punta Arenas & Descarga de aguas servidas en playa \\
\hline
\end{tabular}




\begin{tabular}{|l|l|l|}
\hline & Porvenir & Descarga de aguas servidas en playa \\
\cline { 2 - 3 } & Río Verde & "Mina Invierno" en Isla Riesco. \\
\hline
\end{tabular}

Fuente: Observatorio de Conflictos Ambientales (OLCA). www.justiciambiental.cl y Fundación Terram. www.terram.cl.

Gráfico $\mathbf{n}^{0} 1$ : Relaciones espaciales entre vulnerabilidad social y conflictos ambientales.
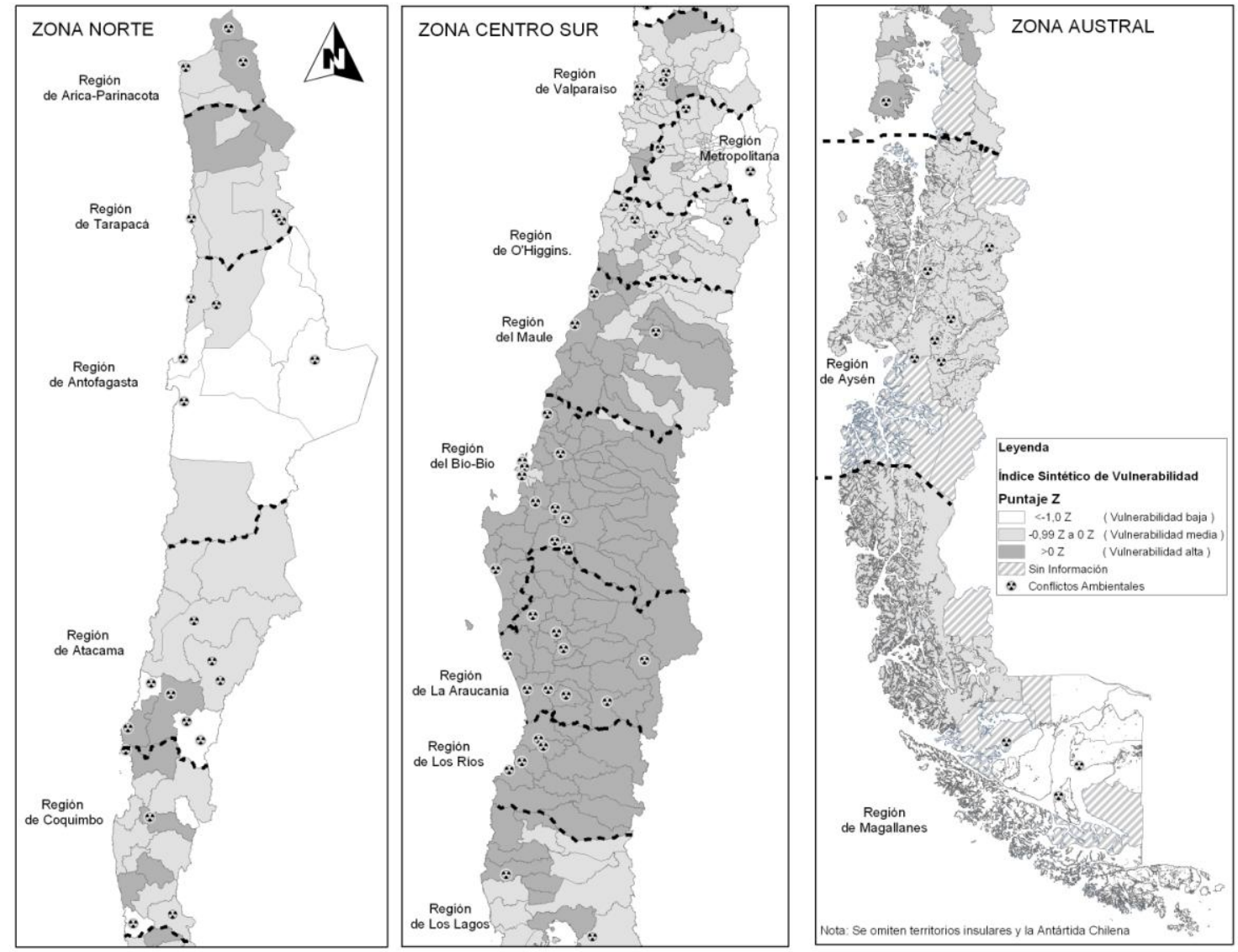

En una segunda instancia, se construye un índice sintético, utilizando información proveniente de la encuesta CASEN 2009, que es un instrumento con representatividad nacional, y que nos permite distinguir los distintitos niveles de vulnerabilidad a escala comunal. Los indicadores utilizados en el índice sintético son aquellos de uso común a nivel latinoamericano. La distribución porcentual de población por pobreza, la tasa de desocupación, el porcentaje de subsidios que componen el ingreso de los hogares, el porcentaje de población inactiva y, el porcentaje de población que finaliza estudios terciarios son, a nuestro juicio, los cinco aspectos de los habitantes de un territorio que mejor definen si una comuna muestra vulnerabilidad social. En teoría, valores altos contenidos en los primeros cuatro indicadores y bajo en el último, determinarían territorios vulnerables para enfrentar un conflicto ambiental por proyectos de inversión privada, en especial si este promete trabajo para la comunidad local. Estadísticamente, de la revisión de la literatura científica nos inclinamos por utilizar el puntaje estándar $(Z)^{1}$, puesto que nos permite realizar comparaciones en la distribución geográfica, a partir de valores con puntajes positivos (alta vulnerabilidad) y negativos (baja vulnerabilidad).

Finalmente, ambos insumos fueron integrados en un SIG de formato vectorial, representando a cada conflicto ambiental como un objeto geográfico tipo punto y cada comuna como un objeto geográfico tipo polígono, permitiendo el procedimiento de análisis espacial a través de la superposición de las dos capas temáticas resultantes (ver gráfico $\mathrm{n}^{\circ} 1$ ). 


\section{El territorio y el conflicto ambiental chileno}

Alguna vez Henri Lefevbre llegó a decir que la única forma de explicar como el capitalismo sobrevivió y se consolidó fue "ocupando espacio" y "produciendo espacio" (Soja, 1980). Pareciera ser una abstracción ortodoxamente geográfica, pero lo cierto es que si improvisamos más activamente la trayectoria del capitalismo mundial podemos darnos cuenta que han sido numerosas las configuraciones de tipo espacial que se desprenden del modelo capitalista. El paso del campo a la ciudad, las nuevas fronteras nacionales venidas de las feudales, o las tensiones internacionales expresadas por el Imperialismo, son solo algunos ejemplos de este silencioso pero fundamental fantasma: el territorio.

Y si decimos silencio no es porque el territorio esté ausente en los estudios y problemas políticos, sociales o económico, sino más bien, detectamos que su uso ocupa un lugar extremadamente estático. El territorio, tal como cuenta Guerevich es la "materialización de los procesos espaciales, es decir, el espacio geográfico puesto en valor, efectivamente usado, tasado o en reserva, donde se contempla el ejercicio del poder político y que resume las relaciones históricas entre la sociedad y la naturaleza" (Gallestegui, 2010: 17). De ahí que no existan azares en las formas organización territorial de los conflictos ambientales, aunque generalmente solo se diga que el territorio es una extensión terrestre delimitada que le da contenido a estos.

Dentro del articulo de los colombianos Montañez y Delgado (2001), destacamos la expresión de territorialidad como expresión y escenario de las relaciones sociales. Entendemos de esta forma que el territorio es un espacio de poder, de gestión y de dominio del Estado, o bien, de individuos, de grupos y organizaciones y de empresas locales, nacionales y multinacionales, pero que evidentemente tanto sus prácticas y usos son diferenciados, ya que cuentan con actores asimétricos y distintos, por tanto, su capacidad de crear, recrear y apropiar el territorio es desigual. En suma, es un producto social, por tanto, implica un conocimiento del proceso de su producción, donde concurren y se sobreponen distintas territorialidades locales, regionales, nacionales y mundiales, con intereses distintos, percepciones, valoraciones diferentes, que generan relaciones de complementación, de cooperación y de conflicto. Por tanto, la dirección de un análisis del territorio del conflicto ambiental chileno, no es fija sino móvil y mutable y desequilibrada, ya que requiere constantemente una organización territorial (Montañez y Delgado, 2001: 122-123).

Así, con estas coordenadas trataremos de ingresar al conflicto ambiental chileno, reconociendo al territorio como una matriz analítica que nos vaya entregando mayores dinámicas y precisiones del movimiento de conflictos ambientales: ¿Cuál es su distribución territorial? ¿En qué tipos de territorios emergen? ¿Qué relación tienen con los escenarios sociales? serán las tres peguntas que nos guiaran en el siguiente análisis.

\section{Discusión}

El análisis del índice sintético de vulnerabilidad nos permite detectar que existe una brecha de 6.11 puntos entre la unidad de análisis que muestra el valor más óptimo, identificada como Vitacura con -3,32Z, y la comuna de Alto Bio Bío con 2,79Z, que ostenta el menos favorable. Esto da cuenta de una acentuada desigualdad en materia de vulnerabilidad social a nivel nacional.

Realizando una valoración a escala comunal (ver cuadro $\mathrm{n}^{\circ}$ 2), podemos subrayar que cerca de un $45 \%$ del territorio administrativo nacional presenta una importante vulnerabilidad, expresado en 149 unidades administrativas. Le siguen 147 comunas con vulnerabilidad media. Solo 38 municipios ostentan buenos indicadores sociales en todas las dimensiones de análisis.

Cuadro no 2: Número de comunas por vulnerabilidad social 


\begin{tabular}{|l|l|l|l|}
\hline$<-1,0 \mathrm{Z}$ & Vulnerabilidad baja & 38 & 11,38 \\
\hline$-0,99 \mathrm{Z}$ a $0 \mathrm{Z}$ & Vulnerabilidad media & 147 & 44,01 \\
\hline$>0 \mathrm{Z}$ & Vulnerabilidad alta & 149 & 44,61 \\
\hline Total & & $\mathbf{3 3 4}$ & $\mathbf{1 0 0}$ \\
\hline
\end{tabular}

Fuente: Elaboración propia.

En consecuencia, su distribución geográfica (ver gráfico ${ }^{\circ} 1$ ), modela un patrón territorial expresado en que la clase de vulnerabilidad alta, ocupa su mayor extensión en la zona centro sur del país, entre las regiones del Maule y de Los Lagos. Asimismo, se presenta en la zona norte, en las comunas pertenecientes a la región de Arica-Parinacota, el norte de la región de Tarapacá y sur de las regiones de Atacama y Coquimbo.

En correspondencia con el objeto central de la investigación, se elaboró el cuadro $n^{\circ} 3$, del que se puede deducir lo siguiente: Las comunas con vulnerabilidad alta poseen el $46,97 \%$ de los conflictos ambientales detectados. Se percibe un evidente escenario de injusticia ambiental, en donde se observa una desigual o desproporcionada participación de grupos de población más desaventajados y, por tanto, más vulnerables, en los impactos negativos resultantes de las actividades mineras, industriales y proyectos de infraestructuras. La desafectación del Parque Nacional Lauca (comuna General Lagos, en la región de AricaParinacota) para la explotación de recursos minerales, y la destrucción del Santuario de la Naturaleza "Carlos Anwandter" (comuna de Valdivia, en la región de Los Ríos) que afectó a cisnes, peces y toda la fauna y flora del río Cruces, producto de la descarga de RILES (residuos industriales líquidos) provenientes de la planta Valdivia de Celulosa Arauco, son ejemplos de lo fácil que es para la inversión extranjera atentar en contra del medio ambiente, aún cuando los territorios estén protegidos por Ley (Sistema Nacional de Áreas Protegidas del Estado). De igual forma, hay que destacar un hecho importante para la temática de investigación: existen 9 conflictos localizados en territorios mapuches (etnia autóctona chilena), lo que nos lleva a reflexionar sobre la posibilidad de existencia de racismo ambiental, que en estos casos en particular, está ligados a vertederos y actividades forestales.

Cuadro nº 3: Número de comunas por vulnerabilidad social

\begin{tabular}{|l|l|l|l|}
\hline INTERVALOS DE CLASE & CATEGORÍA & \multicolumn{1}{l}{$\mathbf{N}^{\mathbf{0}}$ de comunas } & \multicolumn{1}{l}{} \\
\hline$<-1,0 \mathrm{Z}$ & Vulnerabilidad baja & 10 & 15,15 \\
\hline$-0,99 \mathrm{Z}$ a $0 \mathrm{Z}$ & Vulnerabilidad media & 25 & 37,88 \\
\hline$>0 \mathrm{Z}$ & Vulnerabilidad alta & 31 & 46,97 \\
\hline Total & $\mathbf{6 6}$ & $\mathbf{1 0 0}$ \\
\hline
\end{tabular}

Fuente: Elaboración propia. Nota*: *. Se excluyen 2 comunas que presentan conflictos ambientales que no tienen una clasificación de vulnerabilidad social por falta de información.

La vulnerabilidad media contribuye con el $37,88 \%$ de los conflictos ambientales detectados. La individualización de ellos, nos muestra que están ligados a actividades mineras, industriales y agroindustriales. En este grupo de comunas, es interesante destacar las enfermedades provocadas por la contaminación de plomo en barrios pobres, en la comuna de Arica (región de Arica-Parinacota), y las enfermedades respiratorias ocasionadas por fundiciones mineras en Paipote (región de Atacama), en el complejo Quintero-Ventanas y Catemu (ambas en la región de Valparaíso). Tanto en Arica como en Puchuncaví, se demuestra injusticia ambiental al interior de una unidad de análisis, que en un contexto general es favorable, particularmente en las zonas residenciales de Los Industriales y Cerro Chuño para la primera y La Greda, para la segunda.

Por su parte, la categoría vulnerabilidad baja posee el 15,15\% de los conflictos ambientales detectados, ligados a actividades industriales y mineras. El caso más representativo para este grupo de comunas, lo 
constituyen los impactos que se vislumbran para el proyecto minero Pascua Lama, ubicado en la comuna de Alto del Carmen (sur de la región de Atacama); ya que la contaminación de los escasos recursos hídricos de un valle ubicado en zona climática de semiárido (el proyecto original pretendía el desplazamiento de glaciares para utilizar el espacio como depósito de escorias), son ignorados por la población local bajo promesas de trabajo, de aseguramiento de educación universitaria para los individuos en edad escolar y mejora de equipamientos comunitarios. No se puede negar que las medidas compensatorias son suficientes, pero no internalizan los costos que significa eliminar la agricultura y el mayor riesgo de enfermedades (tratamiento y muerte) por el consumo de agua contaminada.

Sin duda alguna, el mercado, aprovecha la vulnerabilidad a la pobreza que tienen los territorios para colocar sin cobro o sin pago las externalidades de los procesos productivos. Que más de un $80 \%$ de los conflictos ambientales estén localizados en territorios de categorías medias y altas es ilustrativo de la dinámica existente en el país. La dimensión espacial de la ecuación vulnerabilidad social + inversión extranjera, en Chile, plasma cambios territoriales que finalmente el Estado debe paliar, acotando su actuación a reaccionar una vez ocurrido un impacto negativo al ambiente, y relegando a un segundo plano la prevención de ellos.

\section{Reflexión}

Las ONG's denuncian que en 68 comunas de Chile existen actualmente conflictos ambientales activos, la mayor parte ligados a la intensificación del modelo exportador primario y su necesidad de energía.

Dado el contexto centralista del Estado chileno, es probable que esta cifra aumente, en especial si se considera el número de proyectos y el volumen de inversión, que espera su ingreso al Servicio de Evaluación Ambiental.

El conflicto aparece cuando en una localidad se pretende instalar un proyecto de inversión extranjera, que cuenta con el patrocinio de una decisión política, sea éste ministerial o presidencial. Esta forma de cultura política, dificulta una participación efectiva de la ciudadanía en la prevención de los conflictos ambientales que presentan sus territorios.

Por tanto, es un verdadero reto para la economía nacional internalizar las externalidades, para que de este modo el modelo de desarrollo adoptado beneficie a todos por iguales y sea más justo ambientalmente, sin importar raza, nivel educacional, ingresos o localización geográfica.

En síntesis, a nivel país observamos que los conflictos ambientales se distribuyen por el largo y ancho del territorio, de manera concentrada a los intereses económicos. Más aún, muestran agrupamientos o cluster en la zona norte ligados a la minería, en el centro a la silvicultura, y en el sur a la generación de hidroelectricidad. Es importante plantear que existe una discriminación social del conflicto ambiental, y por consiguiente, es de preocupación cautelar una proyección social que envuelva el problema ambiental, de manera de promover ideas vinculantes a un mayor y activo compromiso de las comunidades más pobres y sus territorios.

\section{Agradecimientos}

La elaboración de este trabajo se enmarca en el proyecto de investigación DI 037.307/2011, financiado por la Vicerrectoría de Investigación y Estudios Avanzados (VRIEA) de la Pontificia Universidad Católica de Valparaíso.

\section{Notas}

\footnotetext{
${ }^{1}$ Para mayores detalles sobre los procedimientos que se llevan a cabo, consultar la obra de BUZAI, G. (2003): Mapas sociales urbanos, Buenos Aires: Lugar Editorial S.A.
} 


\section{Bibliografía}

Buzai, Gustavo (2003), Mapas sociales urbanos, Lugar Editorial S.A., Buenos Aires.

Gallestegui, Joaquín. y Galea, Juan. (2010), Espacios para una geografía social, humanista y crítica, Ediciones Puntángeles, Universidad de Playa Ancha, Valparaiso.

González, Francisco (2009), “Desarrollo humano sustentable local”, en Polis [online], vol.8, n.22, pp. 53-66.

Harvey, David. (2007), Espacios de Capital: Hacia una Geografía Crítica, Akal, Madrid.

Leff, Enrique (2008), "Decrecimiento o desconstrucción de la economía: Hacia un mundo sustentable", en Polis [online], vol.7, n.21, pp. 81-90.

Montañez, Gustavo y Delgado, Ovidio (1998), "Espacio, Territorio y Región. Conceptos básicos para un proyecto nacional”, en Revista Cuadernos de Geografía, vol. 7, n.2, pp. 120-134.

Naredo, José Manuel (2008), "El conflicto entre eficacia y sostenibilidad: Utilizar el "capital mineral" de la Tierra o el flujo solar y sus derivados renovables", en Polis [online], vol.7, n.21, pp. 91-104.

Soja, Edward (1980), "The socio-spatial dialectic", en Annals of the Association of American Geographers, 70(2), pp. 207-225.

Recibido: 15.09 .2011

Aceptado: 11.03.2012 